Krasimir O. Boyanov*, Mariya A. Choneva, Ivica Dimov, Iliyan V. Dimitrov, Tatyana I. Vlaykova, Fanka D. Gerginska, Slavi D. Delchev, Petar I. Hrischev, Katerina N. Georgieva and Anelia V. Bivolarska

\title{
Effect of oligosaccharides on the antioxidant, lipid and inflammatory profiles of rats with streptozotocin-induced diabetes mellitus
}

https://doi.org/10.1515/znc-2021-0215

Received August 9, 2021; accepted February 4, 2022;

published online February 24, 2022

Abstract: Prebiotics, gut microbiota-fermentable substances, delay the development of type I diabetes. In the present study, we investigated the effect of two prebiotics (galacto-oligosaccharides and xylo-oligosaccharides) on the antioxidant protection, lipid profile, and inflammatory activity of rats with streptozotocin-induced diabetes. The following markers were studied - malondialdehyde, 8-hydroxy-2'-deoxyguanosine, ferric reducing ability of plasma (FRAP), triacylglycerols, total cholesterol (TC), high-density lipoproteins, C-reactive protein (CRP), and interleukin-6. Diabetes was induced in male Wistar experimental rats by streptozotocin injection, while the non-diabetic controls were injected with saline. Afterward the oligosaccharides were administered orally to the experimental animals. The blood collected following the decapitation was analyzed by ELISA. A modified protocol was used only for measuring the FRAP values. The galactooligosaccharides and xylo-oligosaccharides lowered the malondialdehyde levels in the diabetic rats $(p<0.05)$. The galacto-oligosaccharides decreased the serum levels of 8-hydroxy-2'-deoxyguanosine ( $p=0.01)$, while the xylooligosaccharides increased the FRAP $(p<0.05)$ in the experimental animals. None of the oligosaccharides affected

\footnotetext{
*Corresponding author: Krasimir O. Boyanov, Department of Medical Biochemistry, Faculty of Pharmacy, Medical University of Plovdiv, 15A, Vasil Aprilov Blvd., 4002 Plovdiv, Bulgaria,

E-mail: krasimir.boyanov@mu-plovdiv.bg

Mariya A. Choneva, Ivica Dimov, Iliyan V. Dimitrov, Tatyana I. Vlaykova and Anelia V. Bivolarska, Department of Medical Biochemistry, Faculty of Pharmacy, Medical University of Plovdiv, 15A, Vasil Aprilov Blvd., 4002 Plovdiv, Bulgaria

Fanka D. Gerginska and Slavi D. Delchev, Department of Anatomy, Histology and Embryology, Faculty of Medicine, Medical University of Plovdiv, 15A, Vasil Aprilov Blvd., 4002 Plovdiv, Bulgaria

Petar I. Hrischev and Katerina N. Georgieva, Department of Physiology, Faculty of Medicine, Medical University of Plovdiv, 15A, Vasil Aprilov Blvd., 4002 Plovdiv, Bulgaria
}

triacylglycerol and interleukin- 6 concentrations, but the galacto-oligosaccharides decreased the TC and CRP levels in the diabetic animals. Both oligosaccharides exert a beneficial effect on the antioxidant protection of the diabetic rats, but have a minor effect on their lipid and inflammatory profiles.

Keywords: antioxidant protection; diabetes mellitus; galacto-oligosaccharides; rats; xylo-oligosaccharides.

\section{Introduction}

Diabetes mellitus is a group of metabolic disorders characterized by high blood glucose levels (hyperglycemia) resulting from either insufficient production or defects in the secretion of the pancreatic hormone insulin [1]. Apart from the hyperglycemia, other metabolic disorders, such as hyperlipidemia and oxidative stress play a substantial role in the pathogenesis of the disease and put people with diabetes at high risk of developing complications [2]. The non-enzymatic glycation of proteins, spontaneous oxidation of glucose, oxidative stress, and increased lipid peroxidation in diabetes can damage enzymes and cellular processes, which in turn could lead to the development of insulin resistance [1].

A number of human and animal studies have reported about specific prebiotic substrates that exert hypoglycemic effects and thus improve the health of type 1 diabetic patients. Prebiotics are substances that are selectively fermented by the gut microbiota and their products exert a beneficial effect on the health of the host [3]. The favorable effects of prebiotics include selective stimulation of the growth and activity of beneficial intestinal microbes that adequately modulate the immune system of the intestine [4]. Several studies have demonstrated that dietary fibers assist in maintaining microbiota homeostasis by exerting a beneficial effect on the intestinal permeability, thus delaying the development of type 1 diabetes [5-7]. 
The aim of the present study was to investigate the effect of xylo-oligosaccharides (OS1) and galactooligosaccharides (OS2), both commercially available, on biomarkers of the oxidative stress and antioxidant protection, the lipid profile and inflammatory activity in rats with streptozotocin-induced diabetes mellitus. The oxidative stress biomarkers that were studied are malondialdehyde (MDA), 8-hydroxy-2'-deoxyguanosine (8-OHdG) and ferric reducing ability of plasma (FRAP); for the lipid profile - triacylglycerols (TAGs), total cholesterol (TC) and high density lipoproteins (HDL), and the inflammatory biomarkers were C-reactive protein (CRP) and interleukin-6 (IL-6).

\section{Materials and methods}

\subsection{Ethics statement}

This study was performed in strict accordance with the guidelines of the European Community Council directives 86/609/EEC.0.2010/63/ EC. All animal experiments were carried out according to protocols approved by the Bulgarian Agency for Food Safety (BAFS resolution №150/09.04.2019) and are in accordance with the ethical standards of the Medical University of Plovdiv (resolution of the University Ethic Committee №2/13.06.2019).

\subsection{Experimental animals}

The study included 63 male Wistar albino rats (mean body weight $195 \pm 30 \mathrm{~g}$, 8-weeks-old), provided by and bred in the vivarium of the Medical University of Plovdiv. They were randomly divided into six groups: three groups of 12 rats, each with induced diabetes and another three groups of nine rats each used as non-diabetic controls. The animals were housed in cages and kept under standard laboratory conditions: floor area $350 \mathrm{~cm}^{2}$, humidity $55 \pm 10 \%$, temperature $22 \pm 2^{\circ} \mathrm{C}$, a 12 -h light/dark cycle, and free access to food and water for the entire period of the experiment.

The experimental animals were divided into the following six groups:

- Test group DOS1 - diabetic rats, treated with xylooligosaccharides,

- Test group DOS2 - diabetic rats, treated with galactooligosaccharides,

- Positive control group DUT - diabetic rats, untreated (fed a standard diet),

- Test control group NDOS1 - non-diabetic rats, treated with xylooligosaccharides,

- Test control group NDOS2 - non-diabetic rats, treated with galacto-oligosaccharides,

- Negative control group NDUT - non-diabetic rats, untreated (fed a standard diet).

\subsection{Diabetes induction}

Type 1 diabetes was induced in 36 rats (groups DOS1, DOS2, and DUT) that were injected intraperitoneally with a single dose of $60 \mathrm{mg} / \mathrm{kg}$ body weight of streptozotocin (STZ) in freshly prepared citrate buffer (pH 4.5). The remaining 27 animals (control groups NDOS1, NDOS2, and NDUT) were injected with the same volume of saline. All animals were fed a standard diet from birth to the end of the experiment.

\subsection{Treatment of the experimental animals}

Six days after STZ administration, OS1 (xylo-oligosaccharides) was administered orally to 21 animals (experimental group DOS1 and control group NDOS1) and OS2 (galacto-oligosaccharides) - to 21 of the animals (experimental group DOS2 and control group NDOS2). The oligosaccharides were administered at a dose of $100 \mathrm{mg} / \mathrm{kg}$ every day for 9 weeks. Working concentrations of galactooligosaccharides and xylo-oligosaccharides were achieved by dilution in distilled water.

Dietary oligosaccharides are not pure products. They are mixtures of oligosaccharides with different degrees of polymerization (dp) [8]. DP is the number of monomeric units in a macromolecule [9]. The degree of polymerization of the carbohydrates for the two oligosaccharides was as follows: galacto-oligosaccharides (TOS-P from Yakult, Tokyo, Japan) contained 2\% dp2, 48\% dp3, 38\% dp4, 12\% dp5, and xylo-oligosaccharide powder, dp 2-10 (XOS dp 2-10 from Lenzing AG, Lenzing, Austria) contained 99\% XOS - 13\% dp2, 19\% dp3, 11\% dp4, $60 \% \mathrm{dp} \geq 5$.

At the end of week 9, the animals were decapitated after being anesthetised with ketamine/xylazine mixture $(87.5 / 12.5 \mathrm{mg} / \mathrm{kg}$, respectively). After the decapitation the blood was collected, centrifuged at $1500 \mathrm{x} \mathrm{g}$ for $5 \mathrm{~min}$, and the separated serum was frozen at $-18^{\circ} \mathrm{C}$ until the biochemical analysis.

\subsection{Biochemical analyses}

An ELISA method based on standard purchased (commercial) kits was used to determine the biochemical parameters. The following markers were analyzed: malondialdehyde (Rat MDA (Malondialdehyde), ELISA Kit, Wuhan Fine Biotech Co.), 8-hydroxy-2'-deoxyguanosine (8-OHdG (8-hydroxydeoxyguanosine) ELISA Kit, Elabscience Biotechnology Inc.), high density lipoproteins (Rat HDL (High Density Lipoprotein) ELISA Kit, Elabscience Biotechnology Inc.), triglycerides (Triglyceride (Rat) ELISA Kit, BioVision Inc.), total cholesterol (QuickDetect ${ }^{\mathrm{TM}}$ Total cholesterol (Rat) ELISA Kit, MyBiosource, Inc.), C-reactive protein (Rat hs-CRP (high-sensitivity C-Reactive Protein) ELISA Kit, Elabscience Biotechnology Inc.), and interleukin-6 (Rat IL-6 (Interleukin 6) ELISA Kit, Elabscience Biotechnology Inc.).

FRAP was determined using the method of Benzie \& Strain [10]. FRAP reagent was added to the obtained serum, the mixture was incubated for $30 \mathrm{~min}$ in the dark at $37{ }^{\circ} \mathrm{C}$ and the absorbance was measured at $593 \mathrm{~nm}$. The results were expressed as $\mu$ mol Trolox equivalent used to prepare the standard curve. 


\subsection{Statistical analysis}

The statistical analysis was performed with SPSS, v.17 (SPSS Inc., Chicago, IL, USA). The Mann-Whitney $U$-test was used for the comparison of the quantitative variables with non-Gaussian distribution between two independent groups. These variables were presented as median and 95\% confidential interval. Only the FRAP values showed a Gaussian distribution and were therefore presented as mean and standard error of the mean (mean \pm SEM). The comparison was performed with the Independent Samples $t$-Test.

\section{Results}

\subsection{Antioxidant profile}

\subsubsection{MDA}

The oral administration of OS1 to diabetic rats (test group DOS1) resulted in significantly lower levels of MDA compared with those of the positive (DUT) and negative controls (NDUT) by 65 and 64\%, respectively. The most pronounced effect was observed versus the test control group NDOS1 - lower by 77\% (Figure 1).

A similar statistically significant effect of OS2 on the MDA levels was observed in the DOS2 test group. The MDA concentrations were 63 and 62\% lower in comparison with the positive and negative control groups, respectively. OS2 caused a 52\% decrease in the MDA levels of DOS2 group compared with NDOS2 group (Figure 1).
When comparing the effects of OS1 and OS2 given to non-diabetic rats of NDOS1 and NDOS2 groups we found that the MDA levels were 2-fold lower in OS2 versus the effect from OS1 administration $(p<0.05)$ - Figure 1 .

\subsubsection{8-OHdG}

OS2 administration to the diabetic rats (test group DOS2) decreased the serum $8-\mathrm{OHdG}$ levels by $31 \%$ in comparison with the OS2-fed non-diabetic rats (test control group NDOS2) - Table 1. No statistically significant differences

Table 1: Serum levels of 8-OHdG.

\begin{tabular}{lr}
\hline Group & 8-OHdG, $\mathbf{n g} / \mathbf{m L}$, median $(\mathbf{9 5} \% \mathbf{C l})$ \\
\hline DOS1 & $15.22(4.19-26.09)^{\mathrm{a}, \mathrm{b}, \mathrm{c}}$ \\
DOS2 & $16.12(9.94-19.67)^{\mathrm{d}, \mathrm{e}, \mathrm{f}}$ \\
DUT & $16.97(8.77-37.79)$ \\
NDOS1 & $24.09(16.48-36.51)^{\mathrm{g}}$ \\
NDOS2 & $24.71(18.75-38.12)$ \\
NDUT & $17.63(14.12-29.83)$ \\
\hline
\end{tabular}

DOS1 versus DUT ( $(p>0.05)$, DOS1 versus NDOS1 $\left({ }^{b} p>0.05\right)$, DOS1 versus NDUT ( $\left.{ }^{c} p>0.05\right)$, DOS2 versus DUT ( $\left.{ }^{d} p>0.05\right)$, DOS2 versus NDOS2 ( $\left.{ }^{e} p=0.01\right)$, DOS2 versus NDUT $\left({ }^{f} p>0.05\right)$, NDOS1 versus NDOS2 $\left({ }^{g} p>0.05\right)$. 8-OHdG - 8-hydroxy-2'-deoxyguanosine, DOS1 diabetic rats, treated with xylo-oligosaccharides, DOS2 - diabetic rats, treated with galacto-oligosaccharides, DUT - diabetic rats, untreated (fed a standard diet), NDOS1 - non-diabetic rats, treated with xylo-oligosaccharides, NDOS2 - non-diabetic rats, treated with galacto-oligosaccharides, NDUT - non-diabetic rats, untreated (fed a standard diet), $\mathrm{Cl}$ - confidence interval.

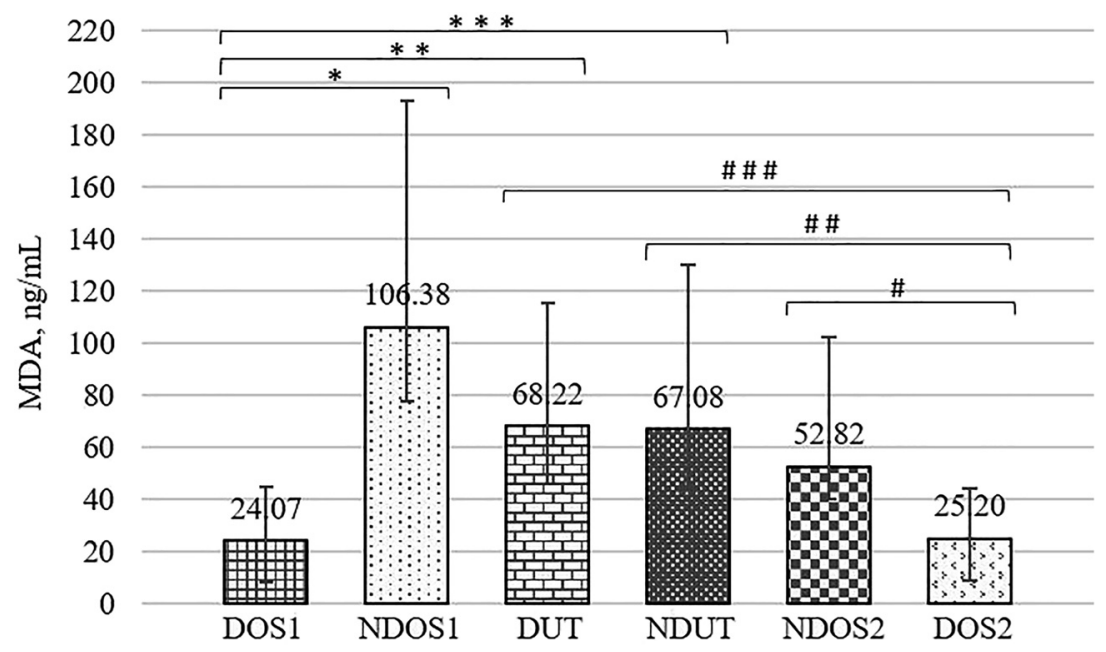

Figure 1: Effects of OS1 and OS2 on serum MDA levels of streptozotocin-induced diabetic rats. Data are expressed as median and $95 \%$ confidential interval. *DOS1 versus NDOS1 $(p<0.01)$; **DOS1 versus DUT $(p<0.01)$; ${ }^{\star \star \star}$ DOS1 versus NDUT $(p=0.025)$; ${ }^{*}$ DOS2 versus NDOS2

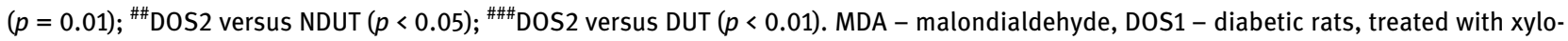
oligosaccharides, DOS2 - diabetic rats, treated with galacto-oligosaccharides, DUT - diabetic rats, untreated (fed a standard diet), NDOS1 non-diabetic rats, treated with xylo-oligosaccharides, NDOS2 - non-diabetic rats, treated with galacto-oligosaccharides, NDUT - non-diabetic rats, untreated (fed a standard diet). 
were found in the 8 -OHdG levels in the other groups (Table 1).

\subsubsection{FRAP}

Administration of OS1 led to significantly higher FRAP levels in the DOS1 test group compared with the positive (DUT) and negative controls (NDUT) by 18 and 16\%, respectively (Figure 2). Furthermore, OS2 tended to increase the FRAP levels in the diabetic rats (test group DOS2) compared to the non-diabetic rats (test control group NDOS2, $p=0.083$ ) - Figure 2. Higher levels of FRAP were found in the DOS1 test group compared with the NDOS1 test control group when OS1 was administered but the values failed to reach statistical significance (Figure 2). Similar results were observed when OS2 was supplied to the DOS2 test group in comparison with the positive (DUT) and negative (NDUT) control groups (Figure 2).

\subsection{Lipid profile}

\subsubsection{TAGs and TC}

The administration of OS1 to the diabetic rats (group DOS1) as well as OS2 to the diabetic and non-diabetic rats (groups DOS2 and NDOS2) significantly elevated (by 524, 492, and $500 \%$, respectively) the serum TAG levels in comparison with the non-diabetic rats fed a standard diet (group
NDUT) - Table 2. On the other hand, treatment of the diabetic rats with OS2 (group DOS2) lowered the levels of the total serum cholesterol by 15\% compared with the levels of the non-diabetic controls (group NDUT) - Table 2. The values for the rest of the groups have no statistically significant differences (Table 2).

\subsubsection{HDL}

When comparing the results of the experiments we conducted with the non-diabetic control groups, we found that the OS1 treated rats had a 9\% higher serum HDL concentration (NDOS1 group) than the rats treated with OS2 (NDOS2 group). Also, HDL tended to be $12 \%$ higher in the healthy rats, in the group that was not treated with oligosaccharides (group NDUT) when compared with the HDL concentration in OS2-treated non-diabetic rats (Table 2). The effect of the two oligosaccharides supplied to the diabetic animals failed to reach statistical significance when compared with that in the controls (Table 2).

\subsection{Inflammatory markers}

\subsection{1 hs-CRP and IL-6}

OS2 administered to the diabetic rats exerted a slightly more beneficial effect on the hs-CRP levels than OS1 did - it reduced hs-CRP by $15 \%$. But hs-CRP concentrations were

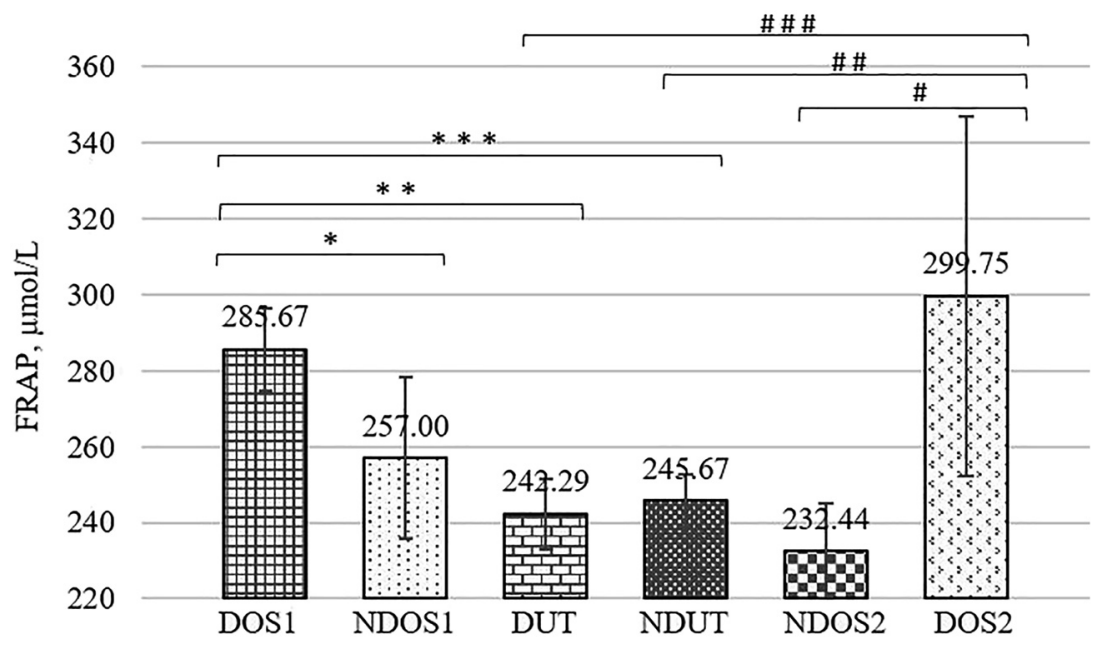

Figure 2: Effects of OS1 and OS2 on serum FRAP levels of rats with streptozotocin-induced diabetes. Data are expressed as mean \pm SEM.

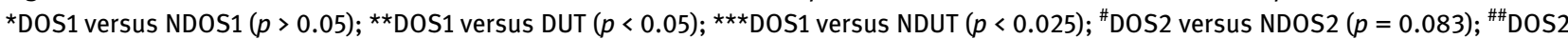
versus NDUT ( $p>0.05)$; \#\#\#DS2 versus DUT $(p>0.05)$. FRAP - ferric reducing ability of plasma, DOS1 - diabetic rats, treated with xylooligosaccharides, DOS2 - diabetic rats, treated with galacto-oligosaccharides, DUT - diabetic rats, untreated (fed a standard diet), NDOS1 non-diabetic rats, treated with xylo-oligosaccharides, NDOS2 - non-diabetic rats, treated with galacto-oligosaccharides, NDUT - non-diabetic rats, untreated (fed a standard diet). 
Table 2: Serum levels of TAGs, TC and HDL.

\begin{tabular}{lrrr}
\hline Group & TAGs, $\boldsymbol{\mu m o l} / \mathbf{L}$, median $(\mathbf{9 5 \%} \mathbf{C l})$ & TC, $\boldsymbol{\mu m o l} / \mathrm{L}$, median $\mathbf{( 9 5 \% ~ C l )}$ & HDL, ng/mL, median (95\% Cl) \\
\hline DOS1 & $1.56(1.27-1.87)^{\mathrm{a}, \mathrm{b}, \mathrm{c}}$ & $1.63(1.06-2.21)^{\mathrm{i}, \mathrm{j}, \mathrm{k}}$ & $5.43(2.64-10.60)^{\mathrm{q}, \mathrm{r}, \mathrm{s}}$ \\
DOS2 & $1.48(1.32-1.70)^{\mathrm{d}, \mathrm{e}, \mathrm{f}}$ & $1.75(1.39-2.06)^{\mathrm{l}, \mathrm{m}, \mathrm{n}}$ & $6.18(-0.25 \text { to } 19.44)^{\mathrm{t}, \mathrm{u}, \mathrm{v}}$ \\
DUT & $1.64(1.51-1.73)$ & $1.85(1.53-2.30)$ & $6.55(5.96-7.47)$ \\
NDOS1 & $1.51(1.32-1.61)^{\mathrm{g}}$ & $1.68(1.48-2.29)^{\mathrm{o}}$ & $5.45(5.28-6.59)^{\mathrm{w}}$ \\
NDOS2 & $1.50(1.36-1.60)^{\mathrm{h}}$ & $1.89(1.63-2.21)^{\mathrm{p}}$ & $5.02(4.87-5.15)^{\mathrm{x}}$ \\
NDUT & $0.25(0.10-1.08)$ & $2.05(1.85-2.54)$ & $5.62(4.82-6.59)$ \\
\hline
\end{tabular}

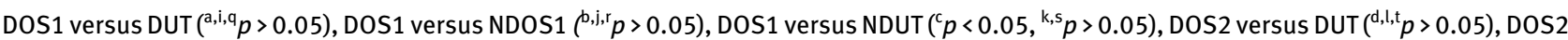
versus NDOS2 (e.m,u $p>0.05)$, DOS2 versus NDUT ( $\left.p=0.007,{ }^{\mathrm{n}} p<0.05,{ }^{\mathrm{v}} p>0.05\right)$, NDOS1 versus NDOS2 $\left({ }^{\mathrm{g}, 0} p>0.05,{ }^{\mathrm{w}} p=0.004\right)$, NDOS2 versus NDUT $\left({ }^{\mathrm{h}} p=0.006,{ }^{\mathrm{p}} p>0.05,{ }^{\mathrm{x}} p=0.085\right)$. TAGs - triacylglycerols, TC - total cholesterol, HDL - high density lipoproteins, DOS1 - diabetic rats, treated with xylo-oligosaccharides, DOS2 - diabetic rats, treated with galacto-oligosaccharides, DUT - diabetic rats, untreated (fed a standard diet), NDOS1 - non-diabetic rats, treated with xylo-oligosaccharides, NDOS2 - non-diabetic rats, treated with galacto-oligosaccharides, NDUT non-diabetic rats, untreated (fed a standard diet), $\mathrm{Cl}$ - confidence interval.

even lower in both control groups treated with OS1 (NDOS1) and fed a standard diet (NDUT) compared with group DOS1 - 23 and 20\%, respectively. On the other hand, OS1 tended to reduce the IL- 6 levels by $48 \%$ in the diabetic rats compared to the non-diabetic ones fed a standard diet (Table 3). No statistically significant differences were observed among the rest of the groups (Table 3).

\section{Discussion}

Hyperglycemia in diabetes is known to cause an increased production of free radicals as a result of protein glycation or glucose autoxidation [11]. The reactive oxygen species (ROS) are directly associated with increased lipid

Table 3: Serum levels of hs-CRP and IL-6.

\begin{tabular}{lrr}
\hline Group & hs-CRP, mg/mL, median & IL-6, pg/mL, median \\
& $(95 \% \mathrm{Cl})$ & $(95 \% \mathrm{Cl})$ \\
\hline DOS1 & $13.27(10.84-15.75)^{\mathrm{a}, \mathrm{b}, \mathrm{c}, \mathrm{d}}$ & $17.63(3.92-32.43)^{\mathrm{j}, \mathrm{k}, \mathrm{l}}$ \\
DOS2 & $11.34(9.27-12.76)^{\mathrm{e}, \mathrm{f}, \mathrm{g}}$ & $25.54(6.26-45.36)^{\mathrm{m}, \mathrm{n}, \mathrm{o}}$ \\
DUT & $11.56(10.25-12.38)$ & $33.98(9.45-54.80)$ \\
NDOS1 & $10.28(9.12-11.01)^{\mathrm{h}}$ & $25.53(17.80-32.73)^{\mathrm{p}}$ \\
NDOS2 & $9.30(8.96-10.68)^{\mathrm{i}}$ & $21.44(18.76-29.31)^{\mathrm{q}}$ \\
NDUT & $10.58(9.22-11.45)$ & $33.98(20.77-40.64)$ \\
\hline
\end{tabular}

DOS1 versus DOS2 ( $\left({ }^{\mathrm{a}} p=0.05\right)$, DOS1 versus DUT $\left({ }^{\mathrm{b}} p=0.089\right.$, $\left.{ }^{\mathrm{i}} p>0.05\right)$, DOS1 versus NDOS1 $\left(p<0.025,{ }^{\mathrm{k}} p>0.05\right)$, DOS1 versus NDUT ( $\left.{ }^{\mathrm{d}} p<0.025,{ }^{\mathrm{l}} p=0.087\right)$, DOS2 versus DUT ( $\left.{ }^{\mathrm{e}, \mathrm{m}} p>0.05\right)$, DOS2 versus NDOS2 $\left({ }^{f, n} p>0.05\right)$, DOS2 versus NDUT $\left({ }^{\mathrm{g}, 0} p>0.05\right)$, NDOS1 versus NDOS2 ( $\left.{ }^{\mathrm{h}, \mathrm{p}} p>0.05\right)$, NDOS2 versus NDUT $\left({ }^{\mathrm{i}, \mathrm{q}} p>0.05\right)$. hs-CRP high-sensitivity C-Reactive Protein, IL-6 - interleukin-6, DOS1 diabetic rats, treated with xylo-oligosaccharides, DOS2 - diabetic rats, treated with galacto-oligosaccharides, DUT - diabetic rats, untreated (fed a standard diet), NDOS1 - non-diabetic rats, treated with xylo-oligosaccharides, NDOS2 - non-diabetic rats, treated with galacto-oligosaccharides, NDUT - non-diabetic rats, untreated (fed a standard diet), $\mathrm{Cl}$ - confidence interval. peroxidation [12,13]. Several studies have shown increased lipid peroxidation in untreated diabetic animals [14-16].

MDA is one of the end products of polyunsaturated fatty acids peroxidation in the cells. Overproduction of MDA is caused by the increase of free radicals [17], which is the reason why MDA is considered as one of the most reliable markers of oxidative stress in clinical practice [18]. MDA levels have been found to be higher in type 2 diabetic patients than those in healthy controls [19]. Furthermore, various authors have reported elevated levels of MDA both in plasma [20] and in some tissues of STZ-induced diabetic rats - in erythrocytes [21], in the pancreas [22], in hepatic tissues [23], in the testicular tissue [24], in the lens [25].

Our results showed that the oral administration of OS1 and OS2 to the diabetic rats (DOS1 and DOS2 groups) reduced the serum MDA levels when compared with the diabetic and non-diabetic groups of rats fed a standard diet (DUT and NDUT) to an almost the same extent. This suggests that galacto-oligosaccharides and xylo-oligosaccharides exert a similar lowering effect on MDA blood levels in the diabetic rats.

Comparing the effect of OS1 and OS2 in the diabetic versus non-diabetic rats, we established that OS2 did not allow a significant increase of the MDA levels in the nondiabetic group (NDOS2), as it was observed in the nondiabetic group treated with OS1 (NDOS1). This observation is also confirmed by the direct comparison of non-diabetic groups treated with OS1 and OS2.

The analysis of our results allowed us to conclude that galacto-oligosaccharides and xylo-oligosaccharides lower the MDA levels of the diabetic rats, with galacto-oligosaccharides being more effective than xylo-oligosaccharides in the nondiabetic rats.

ROS can directly oxidize both the double-stranded DNA and free bases in the cell pools of deoxynucleoside triphosphates [26]. Among all nitrogen bases, guanine is the 
most susceptible to oxidation by ROS [27]. Oxidation of 2'-deoxyguanosine produces 8-hydroxy-2'-deoxyguanosine (8-OHdG). Formation of 8-OHdG in DNA can cause mutations due to improper pairing as a result of the G:C to T:A transversion and these mutations are thought to be closely associated with tumor development and progression, cell aging, and some degenerative diseases [28].

$8-\mathrm{OHdG}$ is one of the products of DNA oxidation that can be easily quantified and is therefore used very often as a biomarker in the oxidative DNA damage assessment [29]. As a marker of oxidative stress, $8-\mathrm{OHdG}$ appears to be in high concentrations in diabetes [30]. This is confirmed by several studies on STZ-induced diabetic rats in which high levels of 8-OHdG were found in the urine [31], in the liver and kidney [32] and in the renal tissues [33].

The results of our experiments show that OS2 treatment beneficially lowers the serum 8-OHdG levels in the diabetic rats compared to the non-diabetic rats. This indicates that the galacto-oligosaccharides not only exert a protective effect against the increasing oxidative stress associated with diabetes, but could also be used for prevention and protection of the cell from the adverse effects of 8-OHdG [28].

FRAP analysis was performed for the antioxidant capacity to be assessed [34]. Several studies have shown that FRAP values in STZ-induced diabetic rats were decreased both in plasma [35] and in various tissues compared to that in controls. The provision of substances that improve the antioxidant status is found to restore FRAP, e.g., curcumin in the liver [36], crab shell extract in the kidneys [37], and Royal jelly in testis [38].

The results demonstrate that OS1 administered to the diabetic rats increases the FRAP values in comparison with the diabetic and non-diabetic rats not treated with an oligosaccharide. The xylo-oligosaccharides are thus suggested to be able to increase the antioxidant capacity in diabetes just like the galacto-oligosaccharides do. The tendency for OS2 treatment to increase the FRAP values in the diabetic rats compared to the non-diabetic rats, further reinforces the effectiveness of the galacto-oligosaccharide as a substance with an antioxidant properties.

Diabetes mellitus is associated with significant abnormalities in the lipid metabolism. Several studies have shown changes in the lipid profile of STZ-induced diabetic rats: elevated serum triacylglycerols levels, total cholesterol and LDL-cholesterol, and decreased HDL levels [39-41].

Our results on serum HDL levels in the non-diabetic groups suggest that OS1 slightly increases the serum HDL levels compared to OS2. Our observation is that oligosaccharide intake has no beneficial effect on the levels of HDL, as a higher level of "good cholesterol" was found in the healthy group not treated with oligosaccharides. The highest HDL concentrations were detected in the diabetic groups (non-significant).

The usefulness of both of the oligosaccharides for treatment of lipid abnormalities is controversial because OS1 and OS2 increase TAG levels in the diabetic (DOS1) and non-diabetic (NDOS2) groups, respectively. Also, OS2 has opposite effects on the serum TAGs and TC levels: in the diabetic rats it significantly increases the concentration of TAGs but slightly lowers the TC levels compared to the nondiabetic rats fed a standard diet. This indicates that the tested galacto-oligosaccharides and xylo-oligosaccharides do not exert a significant effect on the lipid profile in both the control and diabetic rats.

Diabetes mellitus is characterized by a low-grade systemic chronic inflammation [42]. A study has shown a direct link between hyperglycemia, the inflammatory process, and oxidative stress which contribute to the development of chronic diseases [43] but another has reported elevated serum levels of inflammatory mediators such as cytokines and CRP in patients with diabetes [42]. Elevated CRP and IL-6 levels have also been observed in rats with STZ-induced diabetes [44, 45].

In the present study, we demonstrate that OS2 has a stronger significant effect on hs-CRP levels than OS1 in the diabetic rats. Nevertheless, both oligosaccharides have no beneficial effect on the serum $\mathrm{C}$-reactive protein in the diabetic rats because lower amount of hs-CRP is observed in the control groups. On the other hand, OS1 tends to lower IL-6 levels in the diabetic rats compared to the nondiabetic rats fed a standard diet. Further research is needed to study in detail the effect of the two oligosaccharides on inflammation.

\section{Conclusions}

The streamlined design of our experiment in which rats of approximately equal weight were divided into groups (some with successfully induced diabetes and others left as healthy controls) and fed a standard diet with a free access to water, allowed us to test the effects of two commercially available oligosaccharides, OS1 and OS2, on the antioxidant, lipid, and inflammatory profiles.

We report that xylo-oligosaccharides (OS1) and galactooligosaccharides (OS2) exert the most beneficial effect on the antioxidant protection in diabetic rats but have just a minor effect on the various fractions of serum lipids and proinflammatory cytokines.

Further research is warranted for a full examination of the capacity of action of OS1 and OS2 for them to be used in 
the prevention of diabetes mellitus. The antioxidant activity we have established to-date turns them into potential therapeutic agents (to be confirmed by future research) in conditions associated with increased oxidative stress.

\section{Abbreviations}

\begin{tabular}{|c|c|}
\hline OS1 & xylo-oligosaccharides \\
\hline OS2 & galacto-oligosaccharides \\
\hline MDA & malondialdehyde \\
\hline $8-\mathrm{OHdG}$ & 8-hydroxy-2'-deoxyguanosine \\
\hline FRAP & ferric reducing ability of plasma \\
\hline TAGs & triacylglycerols \\
\hline TC & total cholesterol \\
\hline HDL & high-density lipoproteins \\
\hline CRP & C-reactive protein \\
\hline IL-6 & interleukin-6 \\
\hline DOS1 & diabetic rats, treated with xylo-oligosaccharides \\
\hline DOS2 & diabetic rats, treated with galacto-oligosaccharides \\
\hline DUT & diabetic rats, untreated (fed a standard diet) \\
\hline NDOS1 & non-diabetic rats, treated with xylo-oligosaccharides \\
\hline NDOS2 & $\begin{array}{l}\text { non-diabetic rats, treated with galacto- } \\
\text { oligosaccharides }\end{array}$ \\
\hline NDUT & non-diabetic rats, untreated (fed a standard diet) \\
\hline STZ & streptozotocin \\
\hline $\mathrm{DP} / \mathrm{dp}$ & degrees of polymerization \\
\hline ROS & reactive oxygen species \\
\hline DNA & deoxyribonucleic acid \\
\hline LDL & low-density lipoproteins \\
\hline
\end{tabular}

Acknowledgments: We would like to express our gratitude towards the Medical University of Plovdiv for the funding of project № HO-07/2019. A part of the results obtained in the project are presented in this study.

Author contributions: Anelia Bivolarska and Tatyana Vlaykova - conceptualization; Anelia Bivolarska, Tatyana Vlaykova and Katerina Georgieva - methodology; Tatyana Vlaykova - validation; Anelia Bivolarska, Tatyana Vlaykova and Mariya Choneva - formal analysis; Krasimir Boyanov, Ivica Dimov, Iliyan Dimitrov, Fanka Gerginska, Slavi Delchev and Petar Hrischev - investigation; Krasimir Boyanov - writing-original draft preparation; Krasimir Boyanov, Mariya Choneva, Tatyana Vlaykova and Anelia Bivolarska - writing-review and editing; Krasimir Boyanov visualization; Anelia Bivolarska - supervision; Anelia Bivolarska - project administration.

Research funding: This research was funded by Medical University of Plovdiv, Bulgaria, project № HO-07/2019: "Effect of prebiotics and aerobic training on the antioxidant and lipid profiles of rats with streptozotocin-induced diabetes".

Conflict of interest statement: The authors declare no conflict of interest.

\section{References}

1. Maritim AC, Sanders RA, Watkins JB 3rd. Diabetes, oxidative stress, and antioxidants: a review. J Biochem Mol Toxicol 2003; 17:24-38.

2. Kangralkar VA, Patil SD, Bandivadekar RM. Oxidative stress and diabetes: a review. Int J Pharm Appl 2010;1:38-45.

3. Carlson JL, Erickson JM, Lloyd BB, Slavin JL. Health effects and sources of prebiotic dietary fiber. Curr Dev Nutr 2018;2:nzy005.

4. Shokryazdan P, Faseleh Jahromi M, Navidshad B, Liang JB. Effects of prebiotics on immune system and cytokine expression. Med Microbiol Immunol 2017;206:1-9.

5. Xiao L, Van't Land B, Engen PA, Naqib A, Green SJ, Nato A, et al. Human milk oligosaccharides protect against the development of autoimmune diabetes in NOD-mice. Sci Rep 2018;8:3829.

6. Cani PD, Neyrinck AM, Fava F, Knauf C, Burcelin RG, Tuohy KM, et al. Selective increases of bifidobacteria in gut microflora improve high-fat-diet-induced diabetes in mice through a mechanism associated with endotoxaemia. Diabetologia 2007; 50:2374-83.

7. Lau K, Benitez P, Ardissone A, Wilson TD, Collins EL, Lorca G, et al. Inhibition of type 1 diabetes correlated to a Lactobacillus johnsonii N6.2-mediated Th17 bias. J Immunol 2011;186: 3538-46.

8. Shendurse AM, Khedkar CD. Lactose. In: Caballero B, Finglas PM, Toldrá $F$, editors-in-chief. Reference module in food science. Encyclopedia of food and health, 1st ed. London (UK): Elsevier Ltd; 2016, vol. III:509-16.

9. Jenkins AD, Kratochvíl P, Stepto RFT, Suter UW. Glossary of basic terms in polymer science (IUPAC Recommendations 1996). Pure Appl Chem 1996;68:2287-311.

10. Benzie IF, Strain JJ. The ferric reducing ability of plasma (FRAP) as a measure of "antioxidant power": the FRAP assay. Anal Biochem 1996;239:70-6.

11. Wolff SP, Dean RT. Glucose autoxidation and protein modification. The potential role of 'autoxidative glycosylation' in diabetes. Biochem J 1987;245:243-50.

12. Sundaram RK, Bhaskar A, Vijayalingam S, Viswanathan M, Mohan R, Shanmugasundaram KR. Antioxidant status and lipid peroxidation in type II diabetes mellitus with and without complications. Clin Sci (Lond) 1996;90:255-60.

13. Büyükkoçak S, Oztürk HS, Tamer MN, Kaçmaz M, Cimen MY, Durak I. Erythrocyte oxidant/antioxidant status of diabetic patients. J Endocrinol Invest 2000;23:228-30.

14. Gul M, Laaksonen DE, Atalay M, Vider L, Hänninen O. Effects of endurance training on tissue glutathione homeostasis and lipid peroxidation in streptozotocin-induced diabetic rats. Scand J Med Sci Sports 2002;12:163-70.

15. Chen GQ, Lü KR, Yang YQ, Wang S, Bie MJ. [Effects of oxidative stress on MuRF1 expression in skeletal muscle of diabetic rats]. Sichuan Da Xue Xue Bao Yi Xue Ban 2011;42:349-52.

16. Dey P, Saha MR, Chowdhuri SR, Sen A, Sarkar MP, Haldar B, et al. Assessment of anti-diabetic activity of an ethnopharmacological plant Nerium oleander through alloxan induced diabetes in mice. J Ethnopharmacol 2015;161:128-37.

17. Gaweł S, Wardas M, Niedworok E, Wardas P. Dialdehyd malonowy (MDA) jako wskaźnik procesów peroksydacji lipidów w organizmie [Malondialdehyde (MDA) as a lipid peroxidation marker]. Wiad Lek 2004;57:453-5. 
18. Giera M, Lingeman H, Niessen WM. Recent advancements in the LC- and GC-based analysis of malondialdehyde (MDA): a brief overview. Chromatographia 2012;75:433-40.

19. Kesavulu MM, Rao BK, Giri R, Vijaya J, Subramanyam G, Apparao C. Lipid peroxidation and antioxidant enzyme status in Type 2 diabetics with coronary heart disease. Diabetes Res Clin Pract 2001;53:33-9.

20. Ryadinency R, Hadisaputro S, Rachmawati B. Effect of zinc supplementation on triglyceride and malondialdehyde levels: study on diabetic Wistar rats induced with streptozotocin. Med J Indones 2018;27:82-6.

21. Vural H, Sabuncu T, Arslan SO, Aksoy N. Melatonin inhibits lipid peroxidation and stimulates the antioxidant status of diabetic rats. J Pineal Res 2001;31:193-8.

22. Gina L, Aulanni'am A, Mahdi C. MDA and histologic profile of pancreatic diabetic-rats model administered with extract of Glycine max (L.) Merr. J Pure App Chem Res 2016;5:40-7.

23. Sheweita SA, Mashaly S, Newairy AA, Abdou HM, Eweda SM. Changes in oxidative stress and antioxidant enzyme activities in streptozotocin-induced diabetes mellitus in rats: role of Alhagi maurorum extracts. Oxid Med Cell Longev 2016;2016:5264064.

24. Gholizadeh F, Mokarram P, Dastgheib S, Rahpeima Z. The effect of the aquatic extract of stevia on the MDA level and catalase activity in the testicular tissue of streptozotocin-nicotinamideinduced diabetic rats. Shiraz E-Med J 2018;19:e61044.

25. Khorsand M, Akmali M, Akhzari M. Efficacy of melatonin in restoring the antioxidant status in the lens of diabetic rats induced by streptozotocin. J Diabetes Metab Disord 2019;18:543-9.

26. Ichikawa J, Tsuchimoto D, Oka S, Ohno M, Furuichi M, Sakumi K, et al. Oxidation of mitochondrial deoxynucleotide pools by exposure to sodium nitroprusside induces cell death. DNA Repair 2008;7:418-30.

27. Nakabeppu Y, Ohta E, Abolhassani N. MTH1 as a nucleotide pool sanitizing enzyme: friend or foe? Free Radic Biol Med 2017;107: 151-8.

28. Bowerman B. Cell biology. Oxidative stress and cancer: a betacatenin convergence. Science 2005;308:1119-20.

29. Dalle-Donne I, Rossi R, Colombo R, Giustarini D, Milzani A. Biomarkers of oxidative damage in human disease. Clin Chem 2006;52:601-23.

30. Al-Aubaidy HA, Jelinek HF. Oxidative DNA damage and obesity in type 2 diabetes mellitus. Eur J Endocrinol 2011;164:899-904.

31. Kakimoto M, Inoguchi T, Sonta T, Yu HY, Imamura M, Etoh T, et al. Accumulation of 8-hydroxy-2'-deoxyguanosine and mitochondrial DNA deletion in kidney of diabetic rats. Diabetes 2002;51:1588-95.

32. Park KS, Kim JH, Kim MS, Kim JM, Kim SK, Choi JY, et al. Effects of insulin and antioxidant on plasma 8-hydroxyguanine and tissue 8-hydroxydeoxyguanosine in streptozotocin-induced diabetic rats. Diabetes 2001;50:2837-41.
33. Ha H, Kim C, Son Y, Chung MH, Kim KH. DNA damage in the kidneys of diabetic rats exhibiting microalbuminuria. Free Radic Biol Med 1994;16:271-4.

34. Benzie IF, Strain JJ. Ferric reducing/antioxidant power assay: direct measure of total antioxidant activity of biological fluids and modified version for simultaneous measurement of total antioxidant power and ascorbic acid concentration. Methods Enzymol 1999;299:15-27.

35. Cakatay U, Kayali R. The evaluation of altered redox status in plasma and mitochondria of acute and chronic diabetic rats. Clin Biochem 2006;39:907-12.

36. Ghosh S, Bhattacharyya S, Rashid K, Sil PC. Curcumin protects rat liver from streptozotocin-induced diabetic pathophysiology by counteracting reactive oxygen species and inhibiting the activation of $\mathrm{p} 53$ and MAPKs mediated stress response pathways. Toxicol Rep 2015;2:365-76.

37. Ebrahimi Z, Khazaei MR, Ghanbari E, Khazaei M. Renal tissue damages and its antioxidant status improved by crab shell extract in streptozotocin-induced diabetic rat. Adv Biomed Res 2019;8:41.

38. Ghanbari E, Nejati V, Khazaei M. Antioxidant and protective effects of Royal jelly on histopathological changes in testis of diabetic rats. Int J Reprod Biomed 2016;14:519-26.

39. Andallu B, Vinay Kumar AV, Varadacharyulu NCh. Lipid abnormalities in streptozotocin-diabetes: amelioration by Morus indica L. cv Suguna leaves. Int J Diabetes Dev Ctries 2009;29: 123-8.

40. Almeida D, Braga C, Novelli E, Fernandes A. Evaluation of lipid profile and oxidative stress in STZ-induced rats treated with antioxidant vitamin. Braz Arch Biol Technol 2012;55:527-36.

41. Arise R, Aburo O, Farohunbi S, Adewale A. Antidiabetic and antioxidant activities of ethanolic extract of dried flowers of Moringa oleifera in streptozotocin-induced diabetic rats. Acta Fac Med Naissensis 2016;33:259-72.

42. Alexandraki KI, Piperi C, Ziakas PD, Apostolopoulos NV, Makrilakis K, Syriou V, et al. Cytokine secretion in long-standing diabetes mellitus type 1 and 2: associations with low-grade systemic inflammation. J Clin Immunol 2008;28:314-21.

43. de Carvalho Vidigal F, Guedes Cocate P, Gonçalves Pereira L, de Cássia Gonçalves Alfenas R. The role of hyperglycemia in the induction of oxidative stress and inflammatory process. Nutr Hosp 2012;27:1391-8.

44. Ghiasi R, Ghadiri Soufi F, Mohaddes G, Alihemmati A, Somi MH, Ebrahimi $\mathrm{H}$, et al. Influence of regular swimming on serum levels of CRP, IL-6, TNF- $\alpha$ in high-fat diet-induced type 2 diabetic rats. Gen Physiol Biophys 2016;35:469-76.

45. Sunarti S, Nurliyani N, Tyas A, Kristian S, Prasetyastuti P. The influence of goat milk and soybean milk kefir on IL-6 and Crp levels in diabetic rats. Rom J Diabetes Nutr Metab Dis 2015;22: 261-7. 